\title{
The ALICE Detector Control System
}

\author{
P. Chochula, L. Jirden, A. Augustinus, G. de Cataldo, C. Torcato, P. Rosinsky, L. Wallet, M. Boccioli, and \\ L. Cardoso
}

\begin{abstract}
ALICE is one of the six currently installed experiments at the Large Hadron Collider (LHC) at CERN (Geneva, Switzerland). The experiment saw its first particles during the commissioning of the $\mathrm{LHC}$ accelerator in 2008 and is now preparing for the first physics runs foreseen for the autumn of 2009. The experiment is composed of a large number of sub-detectors, each with up to 15 different subsystems that need to be controlled and operated in an efficient and reliable way. For this purpose, a Detector Control System (DCS) has been developed based on latest technologies and using new and innovative approaches. The DCS system has been used with success during the commissioning of the individual detectors as well as during the cosmic runs and the LHC injection tests that were carried out in 2008. This paper gives an overview of the control system and it describes the architecture, the tools and the components that have been used to build it. Examples of technical implementations are given and new trends and techniques used in the system are highlighted.
\end{abstract}

Index Terms-ALICE experiment, CERN, control systems, SCADA.

\section{INTRODUCTION}

A LICE (A Large Ion Collider Experiment) [1] is a generalpurpose, heavy-ion detector designed to study the physics of strongly interacting matter and the quark-gluon plasma in nucleus-nucleus collisions at the CERN Large Hadron Collider (LHC). It is installed in an underground cavern of the $27 \mathrm{~km}$ accelerator ring.

ALICE will allow a comprehensive study of hadrons, electrons, muons, and photons produced in the collision of heavy nuclei $(\mathrm{Pb}-\mathrm{Pb})$, up to the highest multiplicities anticipated at the LHC. The physics programme also includes collisions with lighter ions and at lower energy, in order to vary energy density and interaction volume, as well as dedicated proton-nucleus runs. Data taking during proton-proton runs at the top LHC energy will provide reference data for the heavy-ion programme and address a number of specific strong-interaction topics for which ALICE is complementary to the other LHC experiments.

The ALICE detector, shown in Fig. 1, is composed of 18 sub-detectors, built with the collaboration of about 1000 people from 90 institutes in 30 countries. The overall dimension of the detector is $16 \times 16 \times 26 \mathrm{~m}^{3}$ with a total weight of approximately 10000 tons.

Manuscript received June 18, 2009; revised October 14, 2009. Current version published April 14, 2010.

The authors are with CERN, CH-1211 Geneva 23, Switzerland (e-mail: Peter. Chochula@cern.ch).

Color versions of one or more of the figures in this paper are available online at http://ieeexplore.ieee.org.

Digital Object Identifier 10.1109/TNS.2009.2039944
The ALICE sub-detectors can be grouped into several categories, according to their primary role in the experiment. Tracking is performed by six silicon layers of the Inner Tracking System (ITS) and by the Time Projection Chamber (TPC). The Time of Flight (TOF) array, High Momentum Particle Identification Detector (HMPID) and Transition Radiation Detector (TRD) are main contributors to the particle identification. Spectrometry is covered by the Photon Spectrometer (PHOS) and Electromagnetic Calorimeter (EMCAL). Global features of the reactions are measured by a number of smaller detectors: $\mathrm{T} 0$ provides precise measurement of the collision time, V0 scintillation counters help to reject beam related background. The experiment forward region is covered by the Forward Multiplicity detector FMD, the Photon Multiplicity Detector (PMD) and the compact Zero Degree Calorimeters (ZDC) which are located in the LHC machine tunnel. Finally, the Muon spectrometer consists of the tracking Muon Chambers $(\mathrm{MCH})$ and Muon Trigger Chambers (MTR).

The Detector Control System (DCS), described in this paper, is responsible for safe and correct operation of the ALICE experiment.

The ALICE DCS is built in close collaboration with detector groups in the participating institutes and with controls and technical services groups at CERN. The work of about 100 contributors is coordinated by a small central team at CERN.

The DCS has been designed to assure a high running efficiency by reducing downtime to a minimum. It also maximises the number of readout channels operational at any time, and measures and stores all parameters necessary for efficient analysis of the physics data. The control and monitoring is provided in such a way, that the whole experiment can be operated from a single workspace in the ALICE control room.

The core of the controls system is a commercial Supervisory Control and Data Acquisition (SCADA) system named PVSS [2]. It controls and monitors the detector devices, provides configuration data from the configuration database and archives acquired values in the archival database.

Software framework built on top of PVSS allows for data exchange with external services and systems through a standardized set of interfaces, mostly based on the CERN LHC Data Interchange Protocol (DIP). The DCS subscribes to a large amount of data published by external providers and provides feedback to them. Parameters critical for physics data reconstruction are extracted from the Archival Database and sent to the Offline Conditions database at the end of each run.

The interaction between the DCS and the Trigger, the Data Acquisition System (DAQ) and the High Level Trigger (HLT) is synchronized by the Experiment Control System (ECS). The full context of the ALICE DCS is summarized in Fig. 2. 


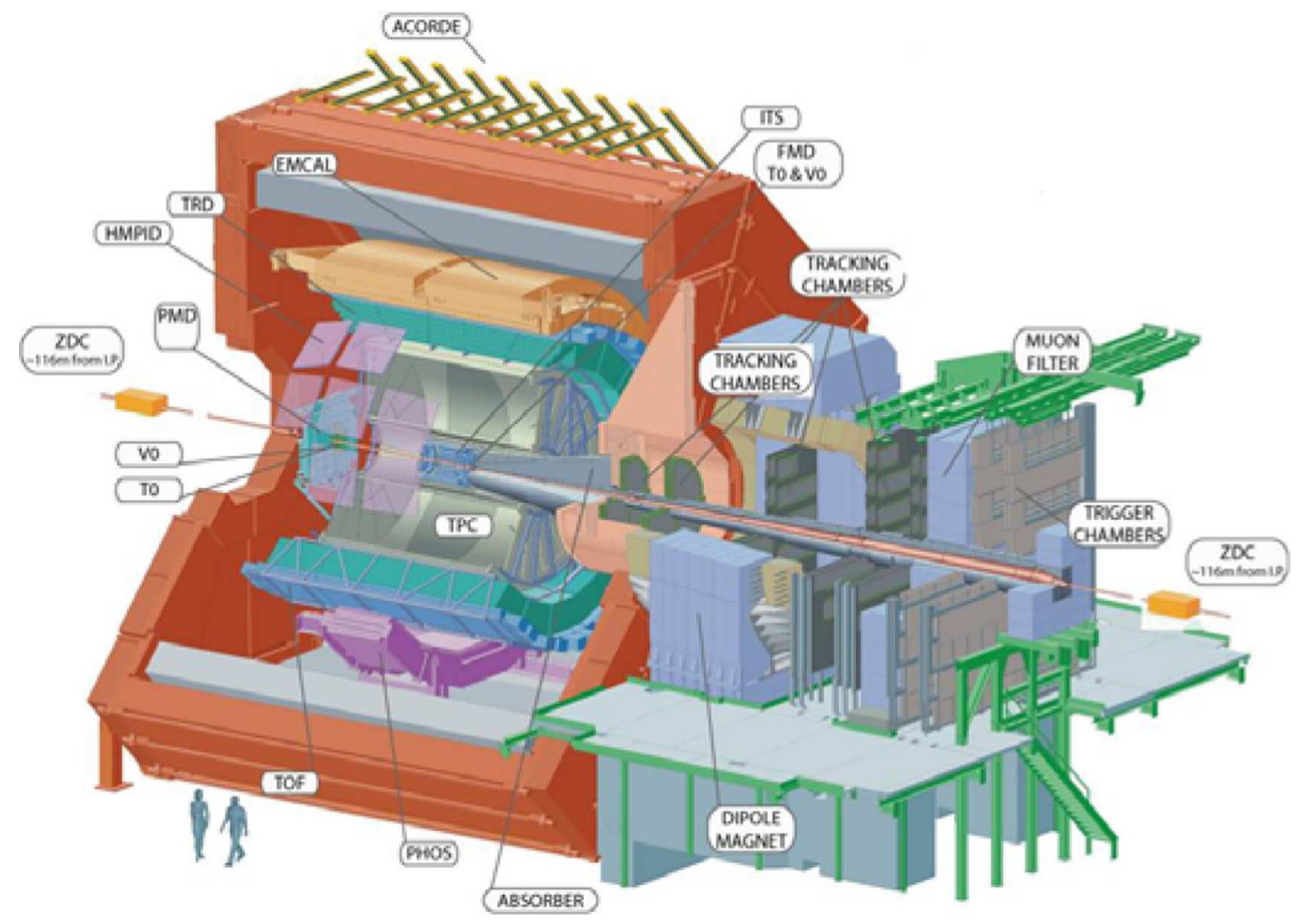

Fig. 1. The ALICE experiment at CERN.

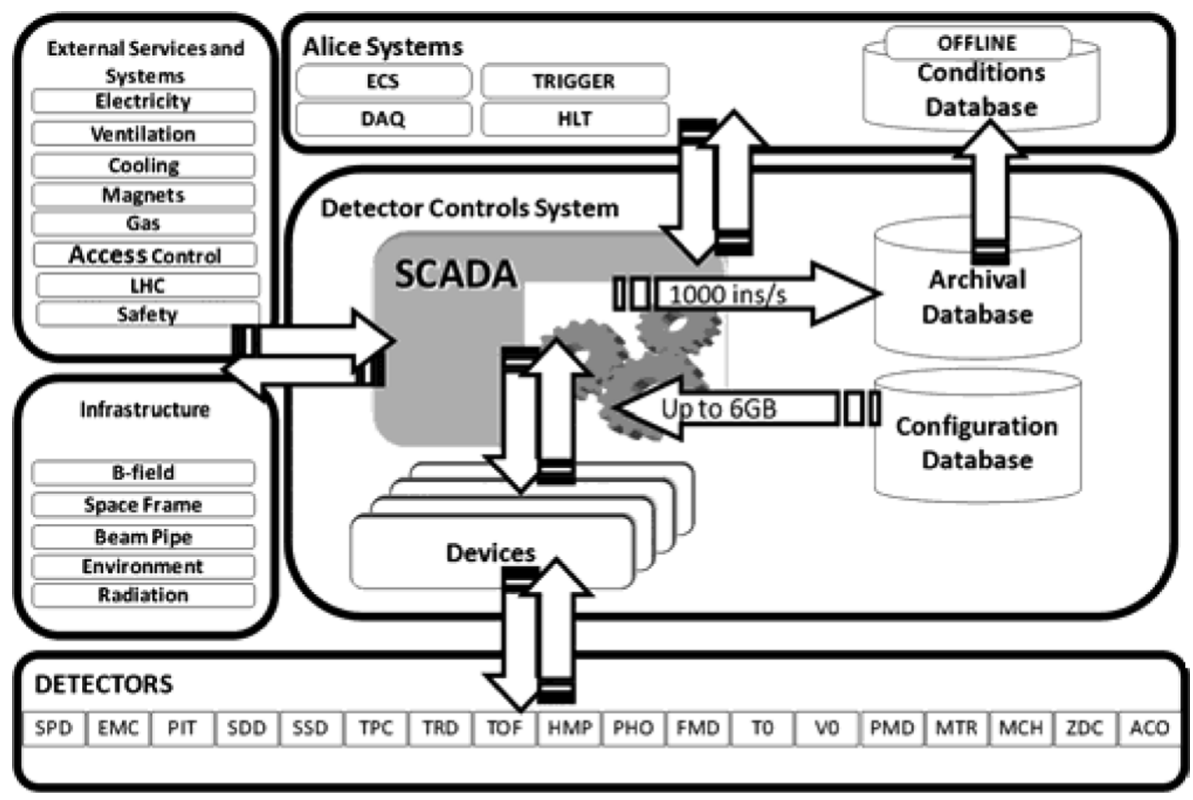

Fig. 2. The DCS context.

\section{Alice DCS ARchitecture}

\section{A. Software Components and Tools}

In order to simplify the design, standardize the communication and complement the PVSS functionalities, a software framework has been built around PVSS. It provides tools and guidelines for easy implementation of the LHC detector control systems. The core of the framework [3] is built as a common effort between the LHC experiments and CERN EN/ICE-SCD section, in the context of a Joint COntrols Project (JCOP) [4]. The main tools cover Finite State Machine (FSM), alarm handling, configuration, archiving, access control, user interfaces, data exchange and communication.

To adopt ALICE's specific needs, the JCOP framework is complemented by ALICE specific components. The complete ALICE framework is used by the sub-detector experts to build their own control applications, such as high voltage control, front-end electronics control, etc. About 140 such applications 


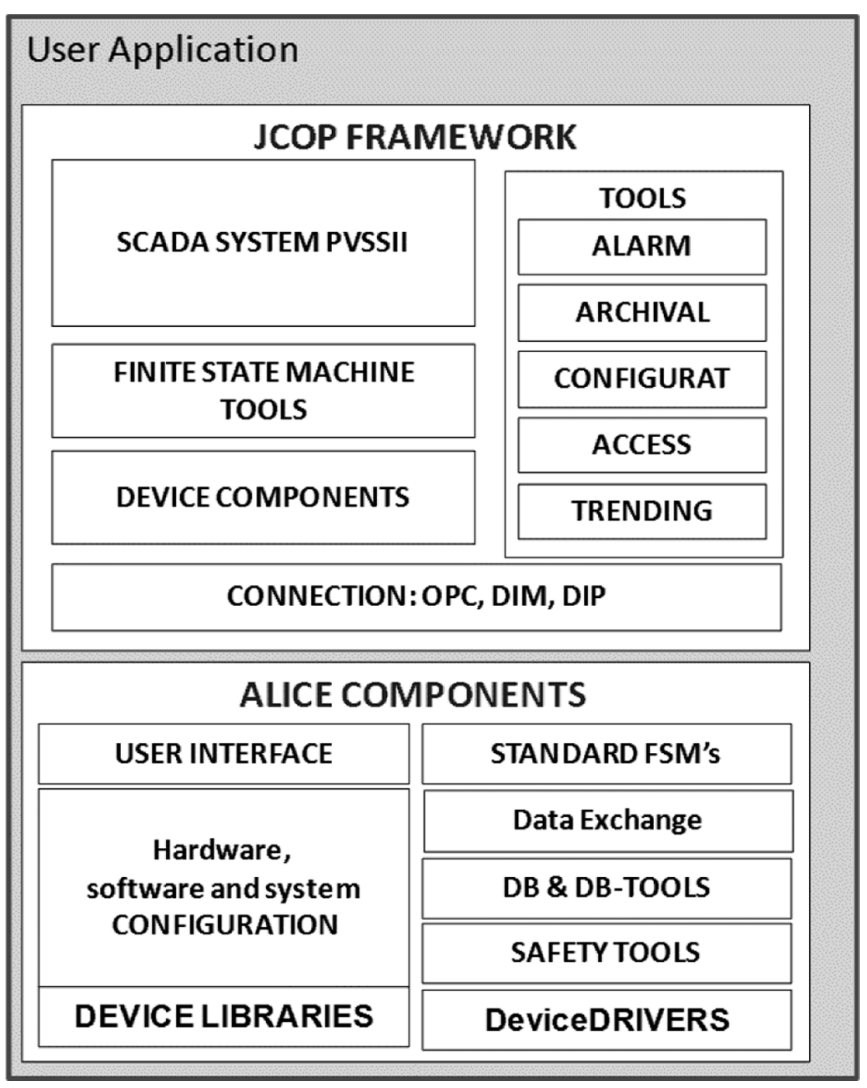

Fig. 3. User application components.

are finally integrated into a large and global ALICE control system. Typical components required to build a user application are summarized in Fig. 3.

\section{B. System Layout}

The whole DCS can be seen as a combination of two planes, a systems plane and an operations plane. The systems plane, shown in Fig. 4, is responsible for the execution of the commands, it assures the communication between the components and it provides the data archival. The systems plane can be further logically subdivided in three layers: a field, a control and a supervisory layer.

The operations plane is a logical layer built on top of the systems plane. It provides a hierarchical representation of the DCS subsystems and it assures the coherent execution of control commands.

\section{The Field Layer}

The field layer consists of devices collecting information from the sub-detectors and providing services to them. Each sub-detector system is logically divided into several sub-systems, each covering different types of devices such as Low Voltage (LV), High Voltage (HV), Front End and Readout Electronics (FEE), etc. There are in total about 150 such sub-systems in ALICE, composed of about 1200 network attached devices and $270 \mathrm{VME}$ and power supply crates. A large effort has been put into the standardization of the hardware used, with the vision to ease the long-term support and service. The OPC protocol has been chosen as standard for the device communication.

The FEE sub-system constitutes a very particular part of the ALICE DCS field layer. Its hardware architecture is detector specific and largely differs from detector to detector. In order to standardize the operation of individual detector FEE, a dedicated access mechanism, called Front End Device (FED) was developed [5]. The FED provides access to the specific FEE hardware of each detector via an abstraction layer having a standardized interface. The communication between the FED and PVSS is carried out by the DIM protocol which is developed at CERN [6]. The FED is also used for devices, which do not provide OPC access.

The scale and complexity of the FEE presents a new challenge for controls systems. For ALICE DCS it means more than 1 000000 FEE channels to control and monitor. To achieve this about 800 single board computers are mounted directly on the detectors. To minimize the network traffic, sampled values are packed into groups, before being sent for processing.

\section{The Controls Layer}

The controls layer consists of $\sim 100$ computers and a number of Programmable Logic Controllers (PLCs) or PLC-like devices, which collect information from the field layer and send control commands to the devices. Most of the computers run PVSS in conjunction with the OPC or FED servers. They rely on infrastructure servers that provide the essential services such as network, storage, database, etc.

As mentioned earlier, the core components of the control layer is PVSS. This is built as a set of managers which communicate via TCP/IP. To operate ALICE about 100 PVSS systems with more than 1000 managers are deployed.

The decentralized PVSS architecture allows for scattering of the systems. In this configuration, the computationally intensive processes run on dedicated hosts in order to balance the load. In addition to this mechanism, PVSS allows for building of distributed systems, where several PVSS systems share data and synchronize the operation of individual managers.

Each detector uses several PVSS systems, each taking care of one or several field layer subsystems. The detector PVSS systems are integrated into one large distributed system. The main ALICE PVSS system is built as a large distributed system of detector distributed systems.

\section{E. The Supervisory Layer}

The computers executing the DCS tasks, called Worker Nodes (WN), typically do not allow for interactive work. The operators use dedicated servers, the Operator Nodes (ON), which provide a set of standardized user interfaces. ALICE ONs form the supervisory layer.

User interfaces executed on the ONs are remotely connected to the individual detector systems on the WNs. This architecture separates the interactive work (like plotting the values) from control tasks and provides a natural protection against the overload of critical systems. Each user interface generating excessive load is automatically blocked. 


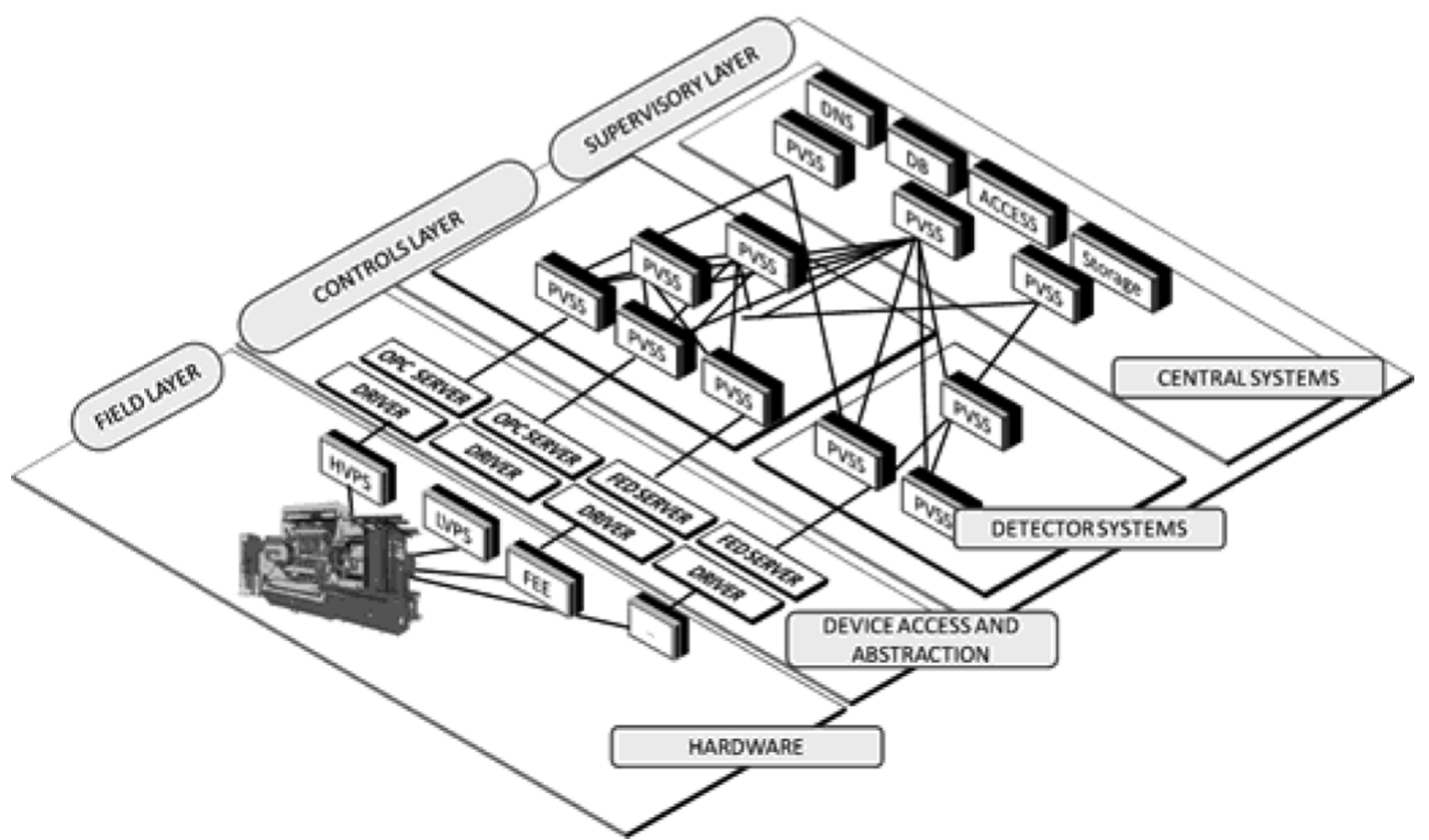

Fig. 4. The ALICE DCS system plane.

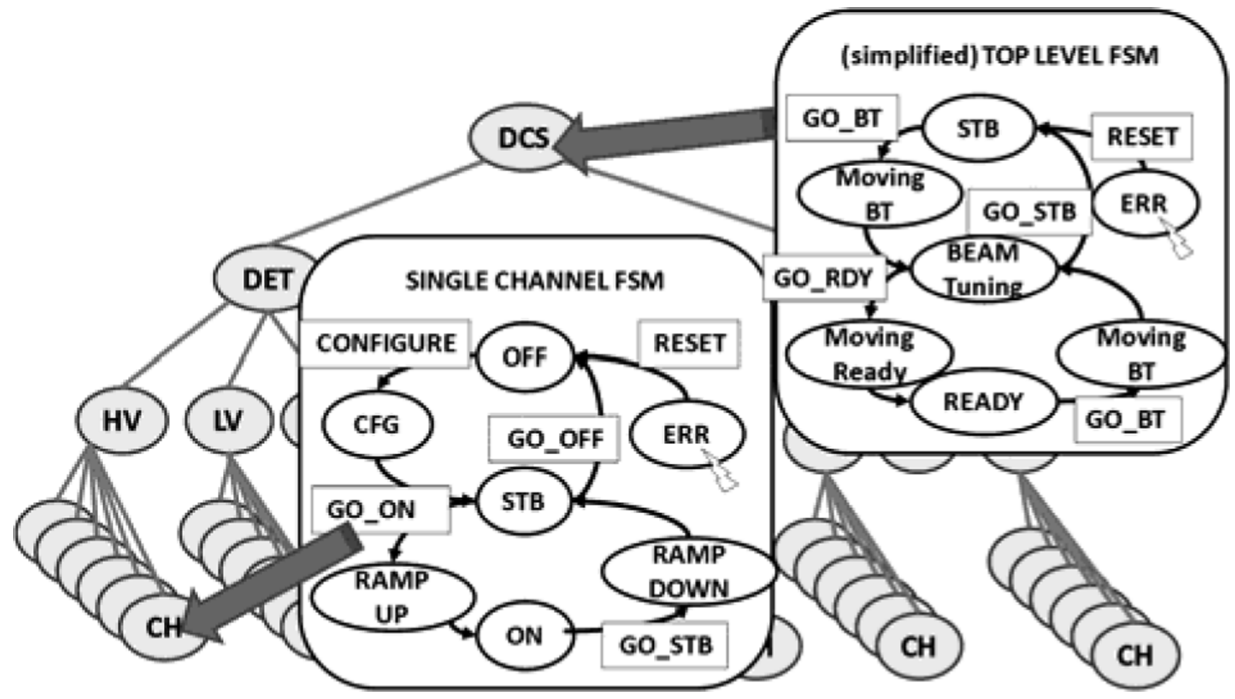

Fig. 5. A simplified picture of the FSM architecture in ALICE.

\section{F. The Finite State Machines}

The behaviour and functionality of each DCS component is described in the terms of FSM using the CERN SMI++ toolkit [7]. Standardized state diagrams deployed in all sub-systems hide the underlying complexity and provide an intuitive representation of the system to be operated.

The FSM represent the operations plane, built on top of the systems plane. Each detector defines a hierarchical structure to represent the layout of the systems to be controlled, starting from channels, device modules up to the complete sub-systems. This tree-like structure is further extended up to the ALICE top-level, which collects information from all sub-systems and sends commands to them.

Each object has a defined set of stable states between which it can transit, as illustrated in Fig. 5. The transition can be either triggered by an operator, or executed automatically, for example as a reaction to an anomaly which requires an intervention. The automatic action can be launched by a command sent by a parent (the object above in the hierarchy), or by state change of the objects below in the hierarchy.

The described architecture allows for automatic and centralized operation of all components. A single operator is able to send commands, which propagate across the tree and execute pre-programmed actions. The set of commands used by the operator is reduced to a minimum, reflecting the ALICE operational needs, such as GO_READY, GO_STANDBY, etc. The FSM mechanism assures that the commands are executed by all targeted leaves and that the actions are synchronized.

The physical execution of the actions is performed by PVSS on the systems plane, and the status is reported back to the oper- 


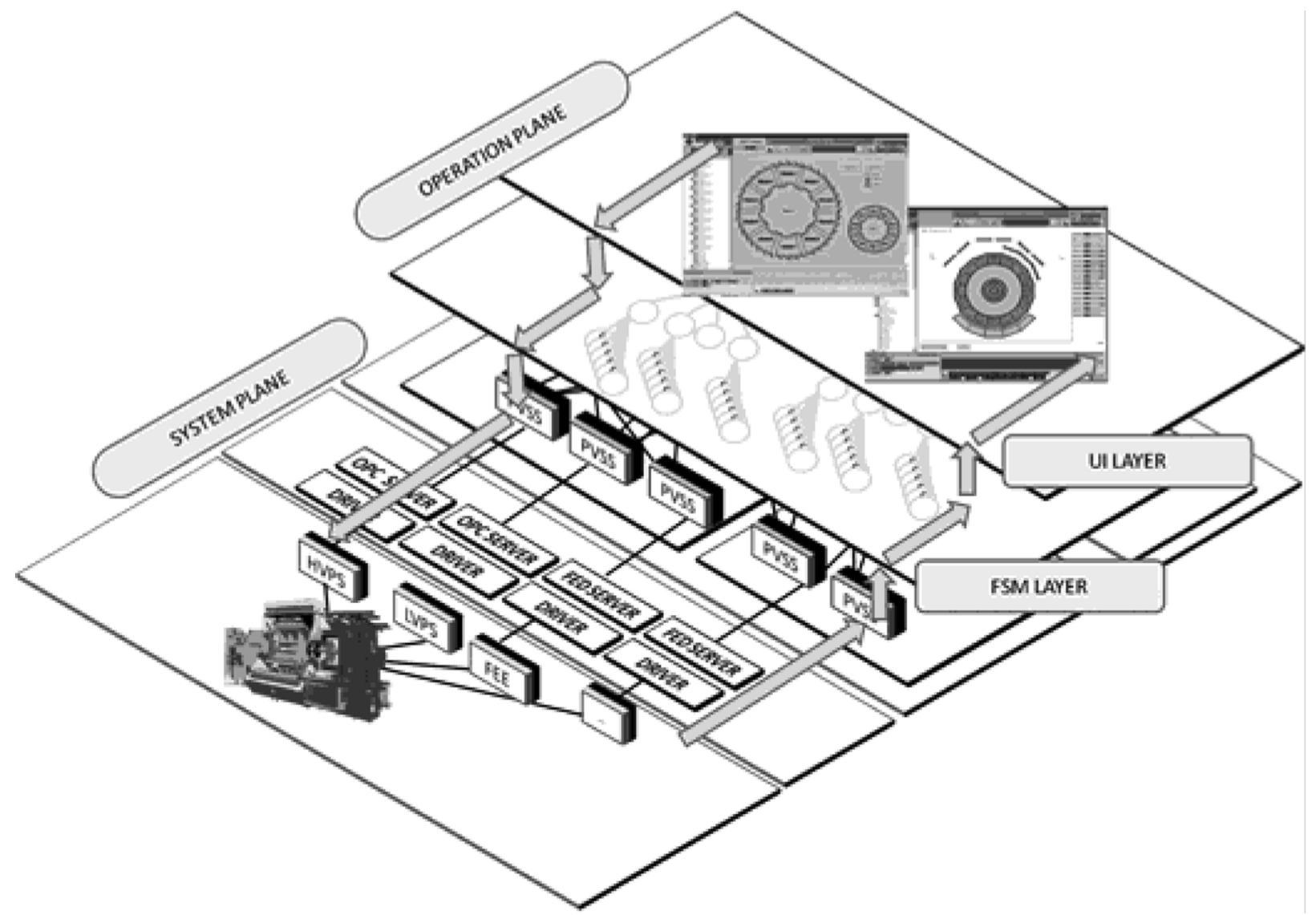

Fig. 6. The FSM and UI layers built on top of the systems plane.

ator via FSM on the operations plane. The global status is computed as a combination of states of all the sub-systems.

\section{G. Partitioning}

A partitioning mechanism implemented by the SMI++ toolkit extends the capabilities of the system and provides additional flexibility. Each individual part of the hierarchical tree can be detached from the main system and operated separately. Thanks to this mechanism, several operators can work in parallel, each operating a different sub-system. In addition, the logical view of the system can be created independently of the systems plane. Masking of hierarchy sub-trees or creation of trees to be operated in separate from the main ALICE tree does not affect the execution of the PVSS systems.

Once a part of the hierarchy is disconnected or masked, its FSM reporting is no longer propagated to the top node. To ensure a safe operation of the experiment, the central operator is nevertheless warned about anomalies from the entire system via the alert mechanism which is implemented on the systems plane. If needed, the central operator can take full control of any sub-tree and perform required tasks.

\section{H. The User Interface}

The Graphics User Interface (GUI) used in ALICE is distributed as a standard component and used by all detectors. The UI represents the top layer in the ALICE DCS architecture, as shown in Fig. 6. The supplied tools and guidelines assure similar look and feel for all components of the DCS. The GUI

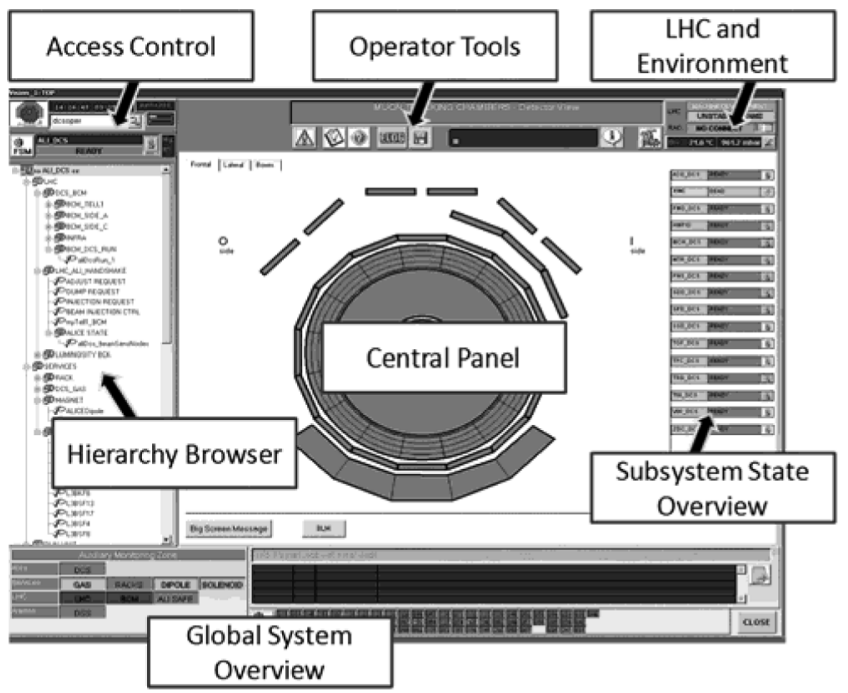

Fig. 7. The ALICE DCS GUIs.

provides hierarchy browser, alert overview, access to FSM and status monitoring. The commands via the GUI are sent to the different components using either the FSM mechanism for standard actions or directly via PVSS for expert actions. Integrated role-based access control mechanism assures protection against inadvertent errors [8].

The GUI exposed to the operators is divided into specific control and monitoring zones and it provides an intuitive overview 


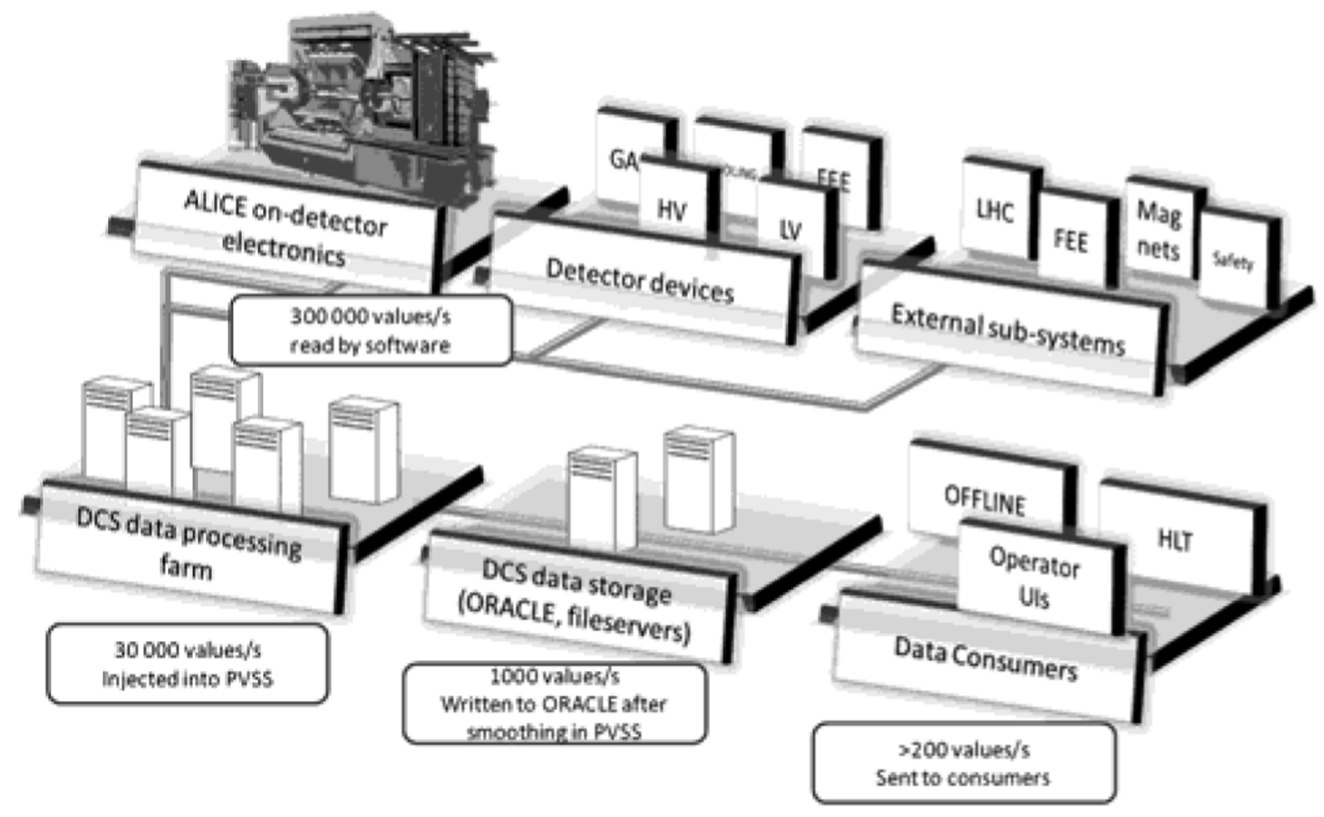

Fig. 8. The ALICE DCS dataflow.

of the system. An example of a typical ALICE DCS UI is given in Fig. 7.

\section{THE Alice DCS DATAFLow}

The amount of data handled by the ALICE DCS goes largely beyond the scale seen in previous generation of control systems [9]. About 1200 network attached devices, $270 \mathrm{VME}$ and power supply crates are needed to provide the infrastructure of the field layer. Up to 6GB of data is loaded from the DCS database to the detector devices at the start of a physics run. This includes PVSS recipes, such as nominal values of device parameters as well as alert limits and FEE settings. One million parameters need to be configured to prepare ALICE for a physics run.

PVSS is constantly monitoring all controlled parameters. About 300000 values are read out by OPC and FED servers each second. To minimize the data traffic, first level filtering is applied at the first level stage and only values exceeding pre-programmed threshold are injected to the PVSS systems. The 10-fold reduction factor is achieved by this mechanism.

Each value processed by the PVSS system if first compared with the nominal one. Should the difference exceed the limit, an alert is generated and displayed on the operator screens. According to the severity an automatic action might be triggered.

All values tagged for archival by the system experts are transferred to the DCS archival database. To reduce the storage requirement, additional level of filtering is applied. Only values falling outside a band defined around the previously archived value are recorded. A new band is then defined around this value. This smoothing mechanism reduces the amount of data written by ALICE DCS to database to about 1000 inserts per second. The six main database servers with total redundant storage of 20TB are configured to cope with a peak insertion rate of 150 000 SQL inserts per second. This is largely sufficient to handle the steady ALICE insertion rate as well as the peak load during the detector configuration and voltage ramping. Full ALICE DCS dataflow is summarized in Fig. 8.

\section{SySTEM COMMISSIONING AND FIRST OPERATIONAL EXPERIENCE}

The controls infrastructure at the experimental site has been installed and is operational since early 2007. This covers all backend infrastructure and common services.

The full ALICE DCS has been integrated and commissioned during 2008 together with the detectors. About 100 individual and common integration sessions were needed to test all functionalities.

The detector integration sessions focused on verification of the functionality of all system components and on compliance with ALICE conventions and rules. Special efforts were given to the detector safety and the commissioning of the interlocks and alerts.

During the common sessions, all participating detectors were operated in parallel. The aim of these sessions was to demonstrate that ALICE DCS can be operated from a single post in the control room. The performance of the backend infrastructure and systems (such as network traffic, archival rates, etc.) was closely studied. Several perturbations were introduced in a controlled way, in order to discover possible irregularities. The acquired experience has been used for further improvements of the systems.

As a result of carefully organized and executed development, integration and test campaigns, the ALICE DCS was fully operational before the LHC startup and contributed to a successful ALICE operation with first beams

\section{REFERENCES}

[1] The ALICE Experiment at the CERN LHC The ALICE Collaboration [Online]. Available: http://www.iop.org/EJ/abstract/1748-0221/3/08/ S08002

[2] Das Objektorientierte Prozessvisualisierungs und Steuerungssystem PVSS [Online]. Available: http://www.pvss.com

[3] Framework Project [Online]. Available: http://itcobe.web.cern.ch/itcobe/Projects/Framework/welcome.html 
[4] G. D. R. Meyers, "The LHC experiments joint control project, JCOP," in Proc. Int. Conf. Accelerator and Large Experimental Physics Control Systems, Trieste, Italy, 1999.

[5] P. Chochula, L. Jirdén, and A. Augustinus, "Control and monitoring of the front-end electronics in ALICE," in Proc. 9th Workshop Electronics for LHC Experiments, Amsterdam, The Netherlands, 2003.

[6] C. Gaspar and M. Donszelmann, "DIM -A distributed information management system for the DELPHI experiment at CERN," in Proc. 8th Conf. Real-Time Computer Applications in Nuclear, Particle and Plasma Physics, Vancouver, BC, Canada, Jun. 1993.
[7] B. Franek and C. Gaspar, "SMI++-An object oriented framework for designing distributed control systems," IEEE Trans. Nucl. Sci., vol. 45, no. 4, pp. 1946-1950, Aug. 1998.

[8] A. Augustinus, P. Chochula, L. Jirden, and P. Rosinsky, "Cyber security in ALICE," in Proc. Int. Conf. Accelerator and Large Experimental Physics Control Systems, Knoxville, TN, 2007.

[9] P. Chochula, A. Augustinus, L. Jirden, S. Kapusta, and P. Rosinsky, "Handling large amounts of data in ALICE," in Proc. Int. Conf. Accelerator and Large Experimental Physics Control Systems, Knoxville, TN, 2007. 